\title{
Neural Correlates of Short-Term Memory Reorganization in Humans with Hippocampal Damage
}

\author{
Carsten Finke, ${ }^{1}$ Hannah Bruehl, ${ }^{2,3}$ Emrah Düzel, ${ }^{5,6}$ Hauke R. Heekeren, ${ }^{2,3,4}$ and Christoph J. Ploner ${ }^{1}$ \\ ${ }^{1}$ Department of Neurology, Charité-Universitätsmedizin Berlin, 10117 Berlin, Germany, ${ }^{2}$ Department of Education and Psychology, ${ }^{3}$ Dahlem Institute for \\ the Neuroimaging of Emotion, and ${ }^{4}$ Cluster of Excellence, Languages of Emotion, Freie Universität Berlin, 14195 Berlin, Germany, ${ }^{5}$ Department of \\ Neurology, University Hospital of Magdeburg, 39120 Magdeburg, Germany, and 'Institute of Cognitive Neuroscience, University College London, London \\ WC1N 3AR, United Kingdom
}

Some patients with disorders affecting the hippocampus have relatively intact memory, but the mechanisms underlying this preservation of function are still debated. In particular, it is unclear whether preserved memory is attributable to significant residual function of unaffected hippocampus or to functional brain reorganization. Here, we investigated brain activation during an associative short-term memory task in two human patient groups matched for extent of postsurgical damage to the right hippocampal formation that differed in two key features, memory performance and preoperative disease course. Patients showed strikingly distinct activation patterns that correlated differentially with behavioral performance, strongly suggesting that intact associative short-term memory with hippocampal dysfunction is indeed related to compensatory brain reorganization. This process appears to depend both on activation of the contralesional hippocampus and on increased engagement of a distributed short-term memory network in neocortex. These data clarify the existence of an efficient hippocampal-neocortical mechanism that compensates for hippocampal dysfunction.

\section{Introduction}

Recent lesion and imaging studies suggest that, beyond its role in long-term memory, the hippocampus is also involved in representation of associative visual information across delays as short as some seconds (for review, see Ranganath, 2010; Jeneson and Squire, 2012). Although observations in patients with focal hippocampal lesions of various etiologies have shown that corresponding short-term memory deficits are clearly present in many subjects (Olson et al., 2006; Hannula et al., 2006; Finke et al., 2008), they were nevertheless absent in others (Braun et al., 2008). Consistent with the central position of the hippocampus in brain networks (van den Heuvel and Sporns, 2011), it has been suggested recently that hippocampal dysfunction may induce structural and functional changes both in the hippocampus and in neocortical regions that are usually linked to working memory functions (Castellanos et al., 2011; Poch and Campo, 2012). However, it is currently unclear whether such network changes indeed mediate compensation of performance in hippocampusdependent memory tasks. So far, imaging studies of resting state functional connectivity in patients with lateralized hippocampal dysfunction have yielded conflicting results, with connectivity

\footnotetext{
Received Feb. 17, 2013; revised April 27, 2013; accepted May 30, 2013.

Author contributions: C.F., H.B., E.D., H.R.H., and C.J.P. designed research; C.F., H.B., and C.J.P. performed research; E.D. and H.R.H. contributed unpublished reagents/analytic tools; C.F., H.B., E.D., H.R.H., and C.J.P. analyzed data; C.F., H.B., E.D., H.R.H., and C.J.P. wrote the paper.

This work was funded by Deutsche Forschungsgemeinschaft Grant PI 248/4-1.

The authors declare no competing financial interests.

Correspondence should be addressed to Carsten Finke, Department of Neurology, Charité-Universitätsmedizin Berlin, Charitéplatz 1, 10117 Berlin, Germany. E-mail: carsten.finke@charite.de.

DOI:10.1523/JNEUROSCI.0744-13.2013

Copyright $\odot 2013$ the authors $\quad 0270-6474 / 13 / 3311061-09 \$ 15.00 / 0$
}

changes in the contralesional hemisphere correlating positively with memory performance in some studies (Bettus et al., 2009) and negatively in others (Campo et al., 2012). In particular, the role of the contralateral hippocampus for compensation of focal lateralized hippocampal damage is controversial. In task-related activation studies using patients with unilateral hippocampal sclerosis, both activation and deactivation of contralateral hippocampus and activation of posterior ipsilateral hippocampus was found to correlate with preserved performance in visuospatial memory tasks (Powell et al., 2007; Figueiredo et al., 2008; Bonelli et al., 2010; Stretton et al., 2012). An open issue is whether these results indeed reflect network reorganization, because in many patient studies, the precise functional status of the diseased hippocampus cannot be determined with certainty. It has moreover proven difficult to disentangle disease-related factors such as active epilepsy clearly from behaviorally relevant compensation of hippocampal injury (Janszky et al., 2004; Figueiredo et al., 2008). Therefore, identification of key mechanisms that drive reorganization may depend critically on research in complementary patient models

Here, we investigated the mechanisms underlying normal memory performance in humans with hippocampal dysfunction by taking a new approach. Task-related brain activity was studied in patients with comparable postsurgical lesions to the right hippocampal formation, but different integrity of memory function. Patients had been treated surgically for either hippocampal sclerosis or benign brain tumor. These two disorders differ significantly in their preoperative disease course, with earlier onset of epilepsy in hippocampal sclerosis patients (Baulac et al., 2004; Sadler, 2006). We have shown previously that this is paralleled by significant performance differences in an associative short-term 
Table 1. Patient data and individual lesion extents

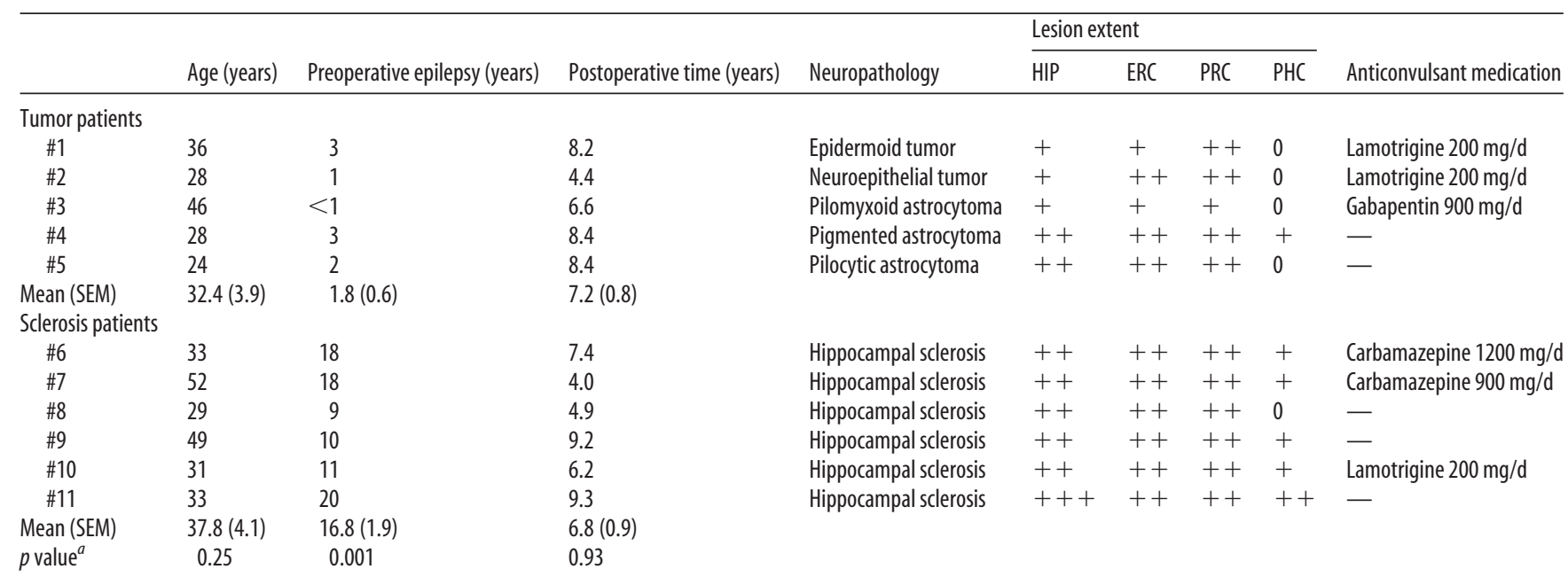

HIP, Hippocampus; ERC, entorhinal cortex; PRC, perirhinal cortex; PHC, parahippocampal cortex; 0 , unaffected subregion; + , rostrocaudal lesion extent of $\leq 20 \mathrm{~mm} ;++$, rostrocaudal lesion extent of $\leq 40 \mathrm{~mm} ;+++$, rostrocaudal lesion extent of $>40 \mathrm{~mm}$.

${ }^{a} p$ values are from group comparisons with Mann-Whitney tests.

memory task sensitive to hippocampal dysfunction, with normal performance in hippocampal sclerosis patients and deficient performance in tumor patients (Braun et al., 2008; Finke et al., 2008). Building on these studies, we compared activation patterns between these patient groups to investigate possible neural correlates of reorganization of associative short-term memory.

\section{Materials and Methods}

Subjects. Eleven patients were recruited from the Department of Neurosurgery and the Department of Neurology at the Charité-Universitätsmedizin Berlin, Berlin, Germany (Table 1). All patients had undergone resection of right medial temporal lobe (MTL) structures for the treatment of epilepsy caused either by a benign brain tumor in the MTL ( 2 females, 3 males, age $32.4 \pm 3.9$ years) or by hippocampal sclerosis ( 3 females, 3 males, age $37.8 \pm 4.1$ years). All patients had already participated in a previous behavioral study by our group (Braun et al., 2008). Histopathological diagnosis was established independently by two neuropathologists who agreed on the diagnosis in each case. Postoperatively, all patients were free of seizures (Engel class I; Engel et al., 1993). All patients were right-handed, free of additional neurological or psychiatric disorders, and normal on neurological examination. All patients were fully integrated into their preoperative social and professional living conditions. By the time of testing, several patients from both groups were taking anticonvulsant medication in regular dosages. All patients were well outside of the postoperative period with similar times since resection (Mann-Whitney test, $p=0.93$; Table 1). In contrast, patients differed significantly with respect to the duration of preoperative epilepsy, with longer disease courses in hippocampal sclerosis patients $(16.8 \pm 1.9$ years) compared with brain tumor patients $(1.8 \pm 0.6$ years; $p<0.001$; Table 1).

The control group consisted of 14 right-handed healthy subjects ( 7 females, 7 males, age $33.0 \pm 1.4$ years) without any history of neurological or psychiatric disorders. There were no differences between the two patient groups and the control group with respect to age (Kruskal-Wallis test, $\mathrm{df}=2, \chi^{2}=1.8, p=0.41$ ) and years of education (tumor patients, $13.4 \pm 0.9$ years; hippocampal sclerosis patients, $15.0 \pm 0.8$ years; control subjects, $14.1 \pm 0.5$ years; $\mathrm{df}=2, \chi^{2}=1.9, p=0.38$ ). Verbal and nonverbal intelligence were assessed in all subjects by the MWT-B, a German equivalent to the National Adult Reading Test (Lehrl, 2005), and the LPS subtest no. 3, a German equivalent to Raven's Progressive Matrices (Horn, 1983). No significant differences in verbal and nonverbal IQ were found between the groups (verbal IQ: tumor patients, $108.6 \pm$ 5.3; hippocampal sclerosis patients, $106.3 \pm 3.6$; control subjects, $109.2 \pm 4.1 ; \mathrm{df}=2, \chi^{2}=0.2, p=0.93$; LPS scores [ $t$-values]: tumor patients, $59.6 \pm 3.5$; hippocampal sclerosis patients, $60.7 \pm 2.6$; control subjects, $62.5 \pm 1.7 ; \mathrm{df}=2, \chi^{2}=0.8, p=0.69$ ).

All participants had normal or corrected-to-normal vision and were native German speakers. The study was approved by the local ethics committee and conducted in conformity with the Declaration of Helsinki. Written informed consent was obtained from all participants.

Lesion analysis. Lesion analysis was performed on high-resolution 3D magnetization prepared rapid acquisition gradient echo (MPRAGE) images that were acquired in all participants (see below). Lesion boundaries were manually delineated on each coronal slice of the individual images using MRIcron software (www.mricro.com/mricron; Rorden et al., 2007). The individual MPRAGE image and lesion shape were then spatially normalized to the MNI brain template using the unified segmentation and normalization approach provided with SPM8 (www.fil.ion.ucl. ac.uk/spm; Ashburner and Friston, 2005). This method has been shown to provide a better and more reliable matching of lesioned brains to a standard template than commonly used alternatives, such as standard nonlinear approaches with cost-function masking (Crinion et al., 2007). Lesion overlap analysis for brain tumor and hippocampal sclerosis patients demonstrated comparable postsurgical lesion extent in both patient groups, with lesions of right anterior hippocampus, amygdala, entorhinal cortex, parts of perirhinal cortex in all patients and additional damage to parahippocampal and inferotemporal cortex in some patients (Fig. 1). In addition, individual lesion extent was determined by using anatomical landmarks proposed by Insausti and Amaral (2004) and Insausti et al. (1998) as described previously (Braun et al., 2008; Table 1).

Stimuli and behavioral paradigms. Visual stimuli were presented using Cogent 2000 (www.vislab.ucl.ac.uk/cogent.php) running in a MATLAB R2010a environment (www.mathworks.com). The stimuli were rearprojected onto a screen inside of the scanner using an LCD projector and viewed through a mirror attached to the head coil. Manual responses were recorded by a two-button response box in the subject's right hand. Subjects performed three variants of a delayed match-to-sample (DMTS) task and a control task with identical visual displays and timing (Fig. 2). The DMTS tasks were modifications of a task that has been shown previously to be sensitive to hippocampal damage and to require short-term memory of colors, locations, or color-location associations (Braun et al., 2008; Finke et al., 2008).

Stimuli were small squares, subtending $0.7 \times 0.7^{\circ}$ of visual angle. In the color and association tasks, squares were of easily discriminable colors, whereas squares in the location task were dark gray. The location of each square was pseudorandomly chosen from 48 possible locations to avoid repetition of sample arrays. The minimal distance between the centers of squares was at least $2.0^{\circ}$. A trial started with the simultaneous 


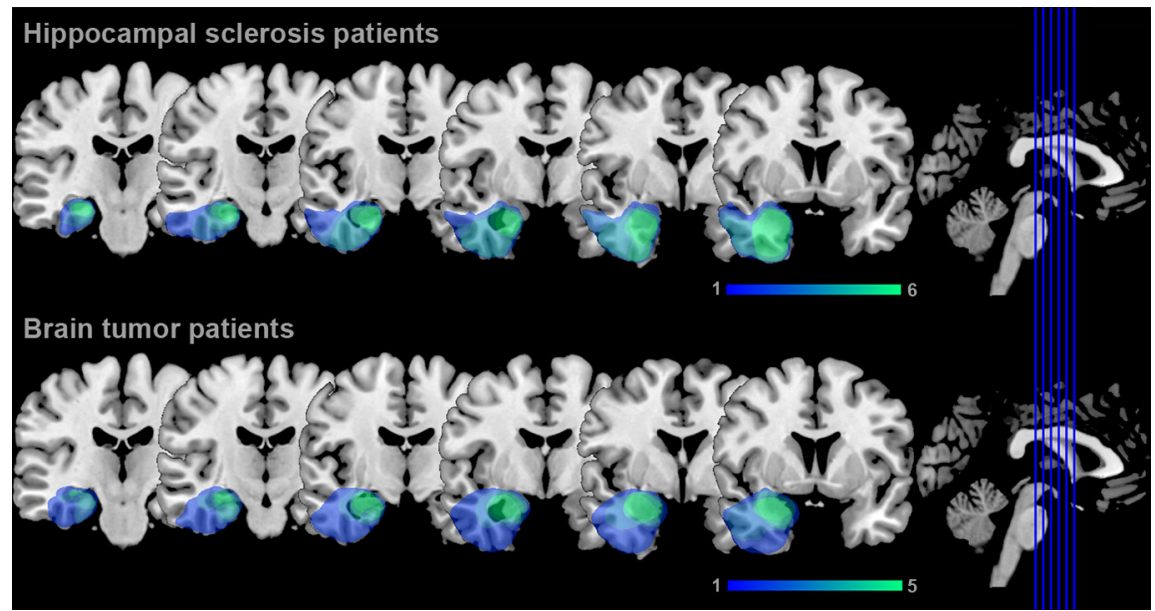

Figure 1. Lesion analysis in patients with postsurgical damage to the right MTL. Lesion overlap analysis depicts right MTL lesions in hippocampal sclerosis and brain tumor patients. Colors indicate the number of patients with lesions affecting a particular voxel.
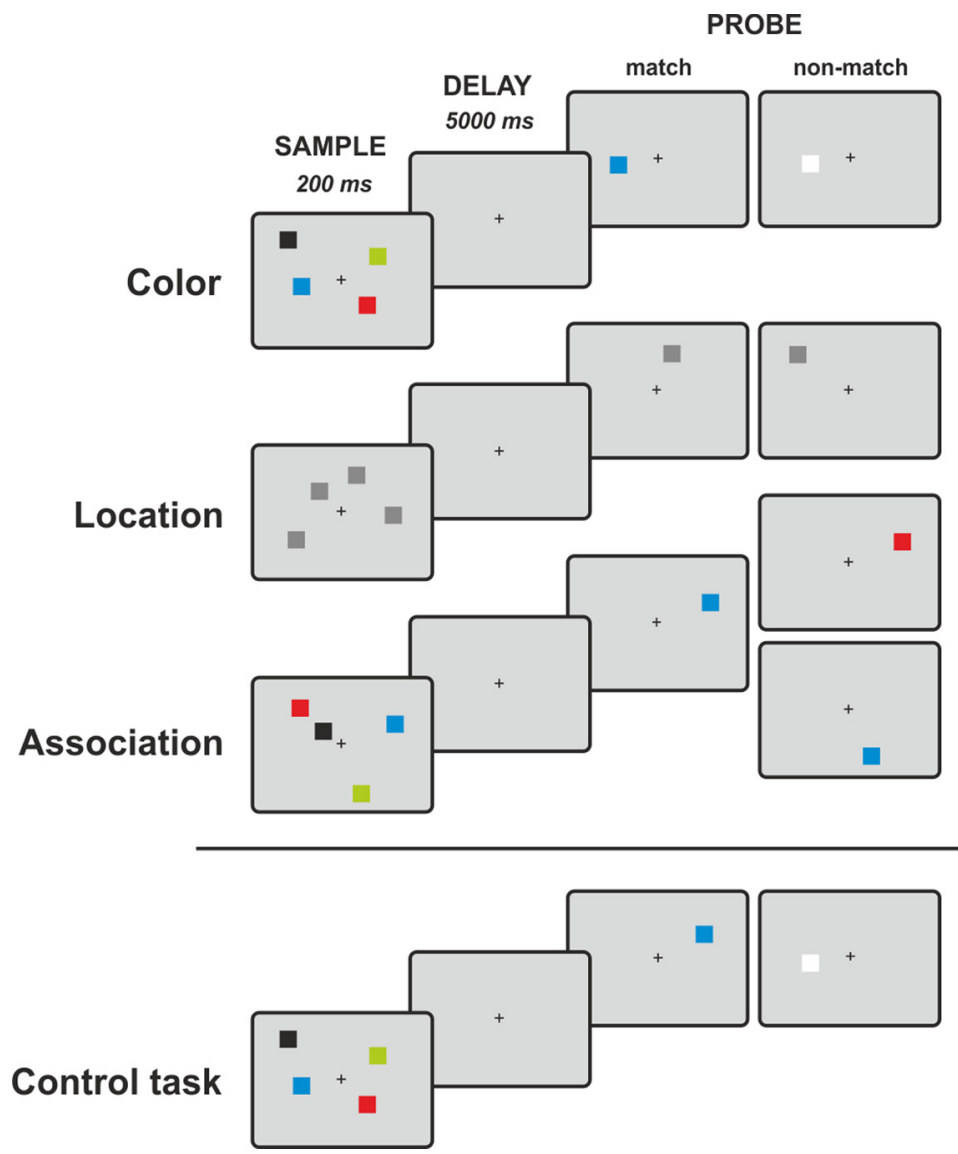

Figure 2. Experimental paradigms. Subjects performed three variants of a delayed match-to-sample task. While fixating a central fixation cross, subjects were presented an array of two or four squares. After a memory delay of $5000 \mathrm{~ms}$, a single probe stimulus appeared and subjects indicated whether the probe matched one of the sample stimuli in color, location, or association (color and location). In the control task, no memory of the sample stimuli was required and subjects instead had to indicate whether the square presented after the delay appeared to the left or right of the fixation dot.

presentation of two or four squares for $200 \mathrm{~ms}$ while subjects fixated on a central dot. After an unfilled delay period of $5000 \mathrm{~ms}$, a probe stimulus appeared and subjects indicated by an unspeeded manual key press whether the probe stimulus matched one of the sample squares in color (color task), location (location task), or color and location (association task). The probe stimulus was presented until the subject pressed a key with a maximum presentation time of $2000 \mathrm{~ms}$; there was no minimum probe presentation time. The length of the intertrial interval was varied between 2 and $14 \mathrm{~s}$ (mean, 5.6; SD, 3.3). The control task required no memory of the sample stimuli. Instead, subjects had to indicate whether the square presented after the delay appeared to the left or right of the fixation dot. The different tasks were administered in separate blocks of eight trials with an equal number of match and nonmatch trials. The experiment comprised four consecutive runs that contained seven experimental blocks in a counterbalanced order. In total, subjects performed 224 trials (i.e., 32 trials per condition). All subjects were given standardized written task instructions and performed two training blocks for each task outside of the scanner.

Task performance was calculated both in percentage correct and $d^{\prime}$ scores (Macmillan and Creelman, 2005). Because we found no differences between results from statistical analyses using either measure of performance, the percentage correct scores are reported henceforth. Because the number of subjects permitted no meaningful conclusions on the normality of the data distribution, nonparametric statistical tests were used (Altman, 1991). Effect sizes were determined by calculating Cohen's $d$ (Friston, 2012).

MRI data acquisition. Whole-brain MRI data were acquired on a Siemens Magnetom Tim Trio 3T scanner equipped with a 12channel phased-array head coil at the Dahlem Institute for Neuroimaging of Emotion at the Freie Universität Berlin, Berlin, Germany. For each subject, four functional runs with $415 \mathrm{vol}$ umes were collected using an echoplanar imaging sequence $(\mathrm{TR}=2000 \mathrm{~ms}$, $\mathrm{TE}=30 \mathrm{~ms}$, flip angle $=70^{\circ}, \mathrm{FOV}=192 \mathrm{~mm} \times 192 \mathrm{~mm}$, matrix size $=64 \times 64$, voxel size $=3 \times 3 \times 3 \mathrm{~mm}$, 37 axial slices aligned to the bicommissural plane, slice thickness $3 \mathrm{~mm}$, interslice gap 0.6 $\mathrm{mm})$. For lesion analysis and registration of the functional images, high-resolution MPRAGE 3D T1-weighted anatomical images were collected $(\mathrm{TR}=1900 \mathrm{~ms}$, TE $=2.52 \mathrm{~ms}$, flip angle $=9^{\circ}$, inversion time $=900 \mathrm{~ms}, \mathrm{FoV}=256$ $\mathrm{mm} \times 256 \mathrm{~mm}$, matrix size $=256 \times 256,176$ sagittal slices, slice thickness $1 \mathrm{~mm}$ ).

fMRI data analysis. MRI data were preprocessed and analyzed using FEAT (fMRI Expert Analysis Tool; version 5.98), which is part of the FMRIB Software Library (FSL; www.fmrib. ox.ac.uk/fsl; Smith et al., 2004). Image preprocessing included brain extraction, slice timing correction, motion correction, spatial smoothing using a $5 \mathrm{~mm}$ FWHM Gaussian kernel, and high-pass filtering (Gaussian-weighted leastsquares straight line fitting, $\sigma=100 \mathrm{~s}$ ) to remove low-frequency noise from the data. FMRIB's Linear Image Registration Tool (Jenkinson and Smith, 2001) was used for a two stage registration of functional images to the MNI space. The functional images were first coregistered to the individual's T1-weighted structural image using a seven-parameter affine transformation and then normalized to the MNI T1 reference brain template provided with FSL using a 12-parameter affine transformation. Analysis of motion parameters revealed no differences between groups (one-way 
ANOVA, absolute motion, $F=0.46, p=0.63$; relative motion, $F=0.41, p=0.96$ ) and absolute head motion was $<1.5 \mathrm{~mm}$ for all subjects (mean, $0.42 \mathrm{~mm}$ ). Statistical analysis was performed within the framework of the general linear model. The time series was modeled individually for each subject and run with eventrelated regressors representing the DMTS tasks (three tasks: color, location, and association $\times$ two loads: two and four items) and the control task. An additional error regressor was used to model brain activity related to incorrect trials. Regressors modeled the whole trial including encoding, maintenance, and retrieval and were convolved with a gamma hemodynamic response function. Contrast images were computed for each task and subject and then entered into a second-level within-subject fixed-effects analysis across runs. Group effects were computed using FMRIB's Local Analysis of Mixed Effects (FLAME) treating subjects as random variable. Given our a priori hypothesis regarding a possible compensatory role of the contralesional hippocampus for associative memory in our patients, an anatomically defined mask was applied to restrict the analysis to the left hippocampus. The mask was defined using the Harvard-Oxford subcortical structural atlas provided with FSL (www. cma.mgh.harvard.edu/fsl_atlas.html) with a $50 \%$ probability threshold. To investigate a possible contribution of neocortical regions, a second, whole-brain group analysis was performed using FLAME. For both analyses, a statistical threshold of $z>2.3$ and correction for multiple comparisons using Gaussian random field theory at cluster level $(p<$ 0.05 ) were used. For simultaneous visualization of significant activations and brain lesions, $z$-maps and group lesion maps were overlaid on a standard anatomical template using MRIcron (www.mricro.com/mricron; Rorden et al., 2007). To further explore differential left hippocampal and neocortical contributions to associative memory between groups, percentage signal change was extracted from regions of interest (ROIs) in the left hippocampus and in the DLPFC and posterior parietal cortex (PPC) bilaterally for each subject. ROIs were defined by clusters of significantly activated voxels from the contrast association task $>$ control task for the comparison of hippocampal scleroses patients $>$ brain tumor patients. Finally, we investigated whether the BOLD change in these ROIs predicted memory performance in the association task on a subject level in patients. To this end, we performed a stepwise linear regression with "percentage signal change" and "patient group" as independent variables and "memory performance" as the dependent variable.

\section{Results}

\section{Behavioral results}

Subjects performed three variants of a delayed match-to-sample task, requiring memory of color, location, or color and location (association) of two or four squares for a delay of $5000 \mathrm{~ms}$ (Fig. 2). Manual response times did not differ between groups (controls, mean $1068 \pm 58$ ms; brain tumor patients, mean $1040 \pm 112 \mathrm{~ms}$; hippocampal sclerosis patients, mean $1011 \pm 83 \mathrm{~ms} ; \mathrm{df}=2, \chi^{2}=$ $0.8, p=0.67$, Kruskal-Wallis test). Memory performance did not differ significantly between groups in the color $\left(\mathrm{df}=2, \chi^{2}=5.2\right.$, $p=0.075)$ and in the location task $\left(\mathrm{df}=2, \chi^{2}=0.4, p=0.815\right.$; Fig. 3). In the association task, however, significant group differ- ences were observed ( $\mathrm{df}=2, \chi^{2}=8.1, p=0.018$ ). Post hoc analyses showed that brain tumor patients performed worse than hippocampal sclerosis patients $(p<0.03)$ and control subjects $(p<0.007)$. Hippocampal sclerosis patients performed at the same level as controls $(p=0.21)$. Calculation of Cohen's $d$ revealed large effect sizes for the observed performance differences between brain tumor patients and controls (Cohen's $d=1.4$ ) and between brain tumor patients and hippocampal sclerosis patients (Cohen's $d=1.13$ ). A small effect size was observed for the difference in performance between controls and hippocampal sclerosis patients (Cohen's $d=0.39$ ). Therefore, we replicated our previous findings that brain tumor patients (i.e., patients with short preoperative disease course) show a selective associative memory deficit, whereas hippocampal sclerosis patients (i.e., patients with long preoperative disease course) had no such memory impairment despite similar lesions of the right MTL (Braun et al., 2008).

\section{fMRI results}

The behavioral results suggest efficient reorganization of memory networks in hippocampal sclerosis patients, but not in brain tumor patients. To investigate whether this compensatory mechanism involves the intact, contralesional hippocampus, we confined our first analysis to the left hippocampus (using an 
Table 2. Left hippocampal ROI analysis results from the contrast of association versus control trials $(z>2.3, p<0.05$, cluster corrected)

\begin{tabular}{llllll}
\hline & & \multicolumn{2}{c}{ MNI coordinates } & & Voxels \\
\cline { 3 - 5 } Group comparison & Region & $x$ & $y$ & $z$ & Z-max \\
\hline Hippocampal sclerosis patients $>$ brain tumor patients & Left hippocampus & -30 & -24 & -18 & 98 \\
Hippocampal sclerosis patients $>$ controls & Left hippocampus & -30 & -22 & -20 & 43 \\
\hline
\end{tabular}
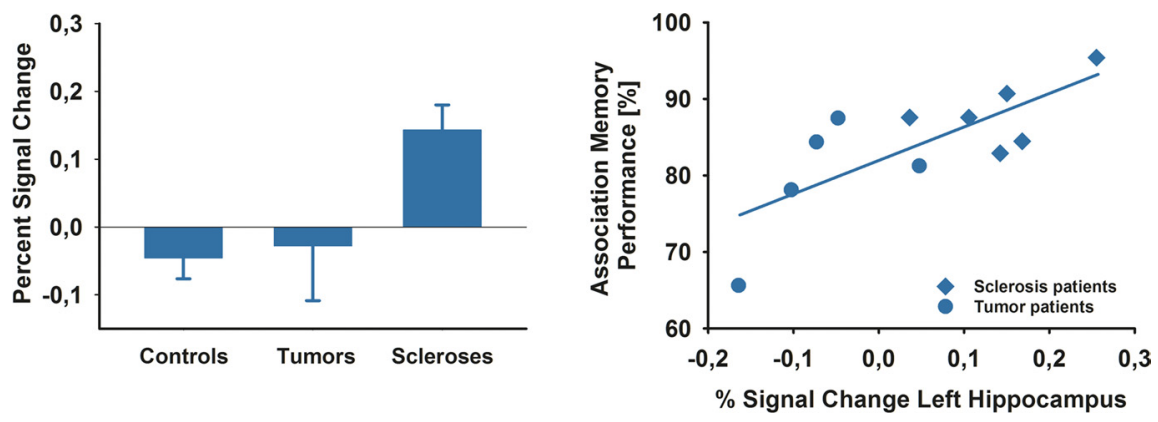

Figure 5. Left hippocampal BOLD signal: percentage signal change and correlation with memory performance. Left, Percentage signal change in the left hippocampal Rol for controls, brain tumor patients, and hippocampal sclerosis patients is plotted for illustrational purposes. Bars represent SEM. Right, Activity in the left hippocampus predicts associative memory performance in patients $(r=0.75, p=0.008)$.

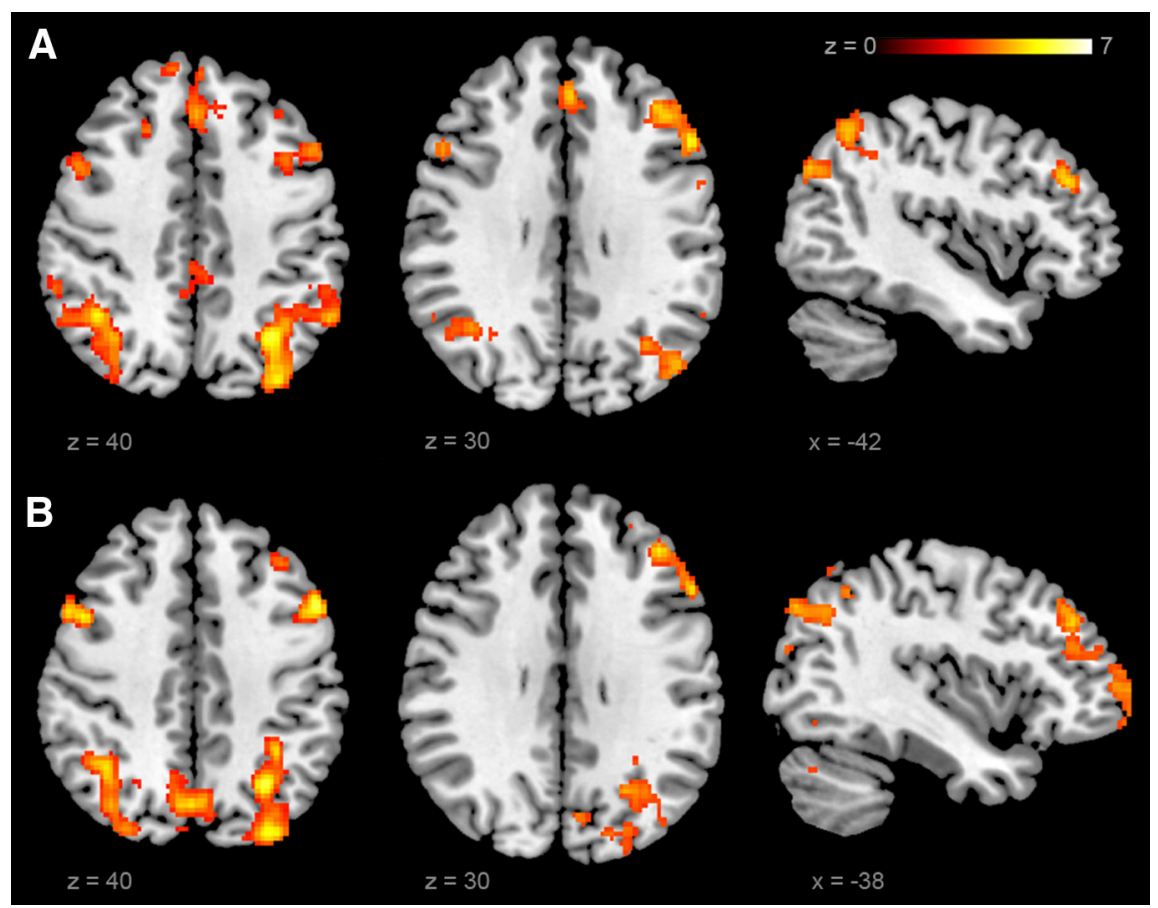

Figure 6. Activation of a neocortical short-term memory network. $\boldsymbol{A}, \boldsymbol{B}$, Neocortical BOLD signal in hippocampal sclerosis patients compared with controls $(\boldsymbol{A})$ and hippocampal sclerosis patients compared with brain tumor patients $(\boldsymbol{B})$ for associative memory trials compared with control trials $(z>2.3, p<0.05$, cluster corrected).

anatomically defined mask, see Materials and Methods). Regarding the performance differences between groups in the association task, we analyzed the BOLD response in association trials in contrast to the BOLD response in control trials. For comparison of hippocampal sclerosis patients with brain tumor patients, we observed a significant cluster of activation in the left hippocampus $(p<0.01$, cluster corrected; Fig. 4, Table 2). Moreover, a similar activation was observed for comparison of hippocampal sclerosis patients with control subjects ( $p<0.01$, cluster corrected; Table 2). No significant activations were found for comparison of brain tumor patients with controls.
To further examine the differential changes in left hippocampal activity between groups, we extracted the mean percentage signal change from the left hippocampal activation (Fig. 5, left). While hippocampal sclerosis patients showed an increase in BOLD signal, brain tumor patients and controls exhibited a slightly reduced BOLD response. Large effect sizes were observed for comparison of percentage signal change between hippocampal sclerosis patients and brain tumor patients (Cohen's $d=1.61$ ) and between hippocampal sclerosis patients and controls (Cohen's $d=1.47)$. In contrast, there was a small effect size for the comparison of controls and tumor patients (Cohen's $d=0.17$ ). Therefore, when comparing patients with controls, we observed a double dissociation in effect sizes for behavioral memory performance and BOLD change in the left hippocampus. In hippocampal sclerosis patients, a small effects size in memory performance (i.e., memory performance at the level of controls) was associated with a large effect size in the percentage signal change analysis of the left hippocampal ROI (increased hippocampal activity compared with controls). The opposite pattern, large effect size for memory performance (i.e., a memory deficit compared with controls) and small effect size of percentage signal change analysis of the left hippocampal ROI (hippocampal activity similar to controls), was found for brain tumor patients.

We also analyzed whether BOLD activity in the left hippocampal ROI predicted performance in the association memory task in patients. Indeed, association memory performance correlated significantly with the percentage signal change in the left hippocampal ROI $(r=0.75,95 \%$ CI $0.27-0.93, p=0.008$; Fig. 5, right). In a stepwise linear regression, the variable group did not enter the model $($ beta $=0.138$, partial correlation $=0.113, p=0.757)$, whereas percentage signal change entered the model and explained $56 \%$ of the variance of memory performance $\left(r^{2}=0.56\right.$, $p=0.008)$.

To examine possible compensatory contributions of neocortical brain regions, we performed a whole-brain analysis for the contrast association $>$ control task. We found a greater BOLD response for hippocampal sclerosis patients compared with brain tumor patients bilaterally in the DLPFC, in the inferior temporal cortex, and in the PPC extending into the precuneus bilaterally. 
Table 3. Whole-brain analysis results from the contrast of association versus control trials $(z>2.3, p<0.05$, cluster corrected)

\begin{tabular}{|c|c|c|c|c|c|c|}
\hline \multirow[b]{2}{*}{ Group comparison } & \multirow[b]{2}{*}{ Region } & \multicolumn{3}{|c|}{ MNI coordinates } & \multirow[b]{2}{*}{ Voxels } & \multirow[b]{2}{*}{ Z-max } \\
\hline & & $x$ & $y$ & $z$ & & \\
\hline \multirow[t]{11}{*}{ Hippocampal sclerosis patients $>$ brain tumor patients } & Precuneus & 0 & -66 & 54 & \multirow[t]{3}{*}{$3783^{a}$} & 6.78 \\
\hline & Left posterior parietal cortex & -30 & -62 & 40 & & 5.71 \\
\hline & Right posterior parietal cortex & 34 & -62 & 48 & & 5.58 \\
\hline & Left thalamus & -6 & -10 & 8 & 790 & 4.95 \\
\hline & \multirow[t]{2}{*}{ Left middle frontal gyrus } & -52 & 14 & 40 & 736 & 5.82 \\
\hline & & -30 & 58 & -2 & 556 & 4.64 \\
\hline & \multirow[t]{2}{*}{ Right middle frontal gyrus } & 48 & 10 & 40 & 136 & 5.29 \\
\hline & & 44 & 54 & -4 & 392 & 5.5 \\
\hline & Left inferior temporal gyrus & -48 & -62 & -16 & 300 & 4.51 \\
\hline & Right inferior temporal gyrus & 52 & -64 & -6 & 272 & 4.46 \\
\hline & Left fusiform gyrus & -28 & -68 & -16 & 205 & 3.31 \\
\hline \multirow[t]{10}{*}{ Hippocampal sclerosis patients $>$ controls } & Left posterior parietal cortex & -32 & -70 & 48 & 1338 & 6.67 \\
\hline & Right posterior parietal cortex & 42 & -54 & 38 & 1031 & 5.55 \\
\hline & \multirow[t]{2}{*}{ Left middle frontal gyrus } & -48 & 36 & 26 & 570 & 5.64 \\
\hline & & -36 & 10 & 54 & 94 & 4.36 \\
\hline & \multirow[t]{2}{*}{ Right middle frontal gyrus } & 50 & 20 & 26 & 157 & 4.9 \\
\hline & & 46 & 42 & 16 & 107 & 4.87 \\
\hline & \multirow[t]{2}{*}{ Left superior frontal gyrus } & -28 & 64 & -6 & 166 & 4.4 \\
\hline & & -14 & 36 & 50 & 120 & 5.11 \\
\hline & Right superior frontal gyrus & 28 & 24 & 50 & 106 & 4.43 \\
\hline & Left + right medial frontal gyrus & -2 & 40 & 32 & 325 & 5.09 \\
\hline
\end{tabular}

${ }^{a}$ Number of voxels listed for Precuneus includes the number of voxels for Left and Right posterior parietal cortex.

We also observed a greater change in BOLD signal of the left thalamus. Activations were pronounced in the left hemisphere, with larger cluster sizes and greater $z$-values (Fig. 6, Table 3). A similar pattern of a bilateral but left-lateralized network was observed for the contrast of hippocampal sclerosis patients and controls, with bilateral activations in the DLPFC, the superior frontal gyrus, the medial frontal gyrus, and the PPC (Fig. 6, Table 3). Percentage signal change analyses revealed a different pattern in comparison to hippocampal activity. Hippocampal sclerosis patients showed a larger BOLD signal increase than controls, whereas in brain tumor patients a reduced BOLD signal was observed (Fig. 7). Performance in the association memory tasks correlated significantly with percentage signal change in the left DLPFC ROI $(r=0.46$, 95\% CI 0.0670.726, $p=0.025)$. In a stepwise linear regression analysis, group did not enter the model ( $p=0.28)$, whereas percentage signal change explained $21 \%$ of the variance $\left(r^{2}=0.21, p=0.025\right)$.

\section{Discussion}

We investigated the mechanisms mediating normal associative short-term memory performance in human subjects with damage to the right hippocampal formation. Two patient groups with similar surgical treatment, lesion extent, and time since resection were studied. However, groups differed significantly with respect to their performance in an associative short-term memory task that has been shown previously to depend on integrity of the right hippocampus (Braun et al., 2008; Finke et al., 2008). In fMRI, differential activation patterns were observed between patient groups, strongly suggesting that successful compensation of uni-
Left DLPFC
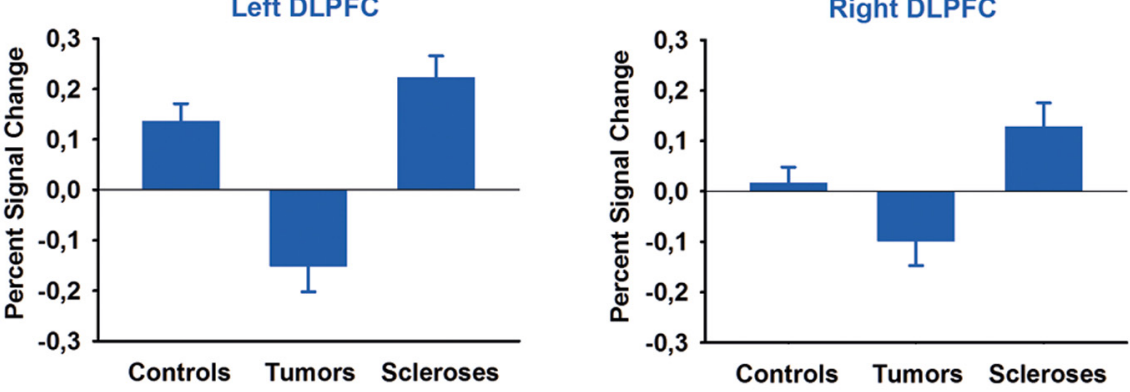

Left PPC
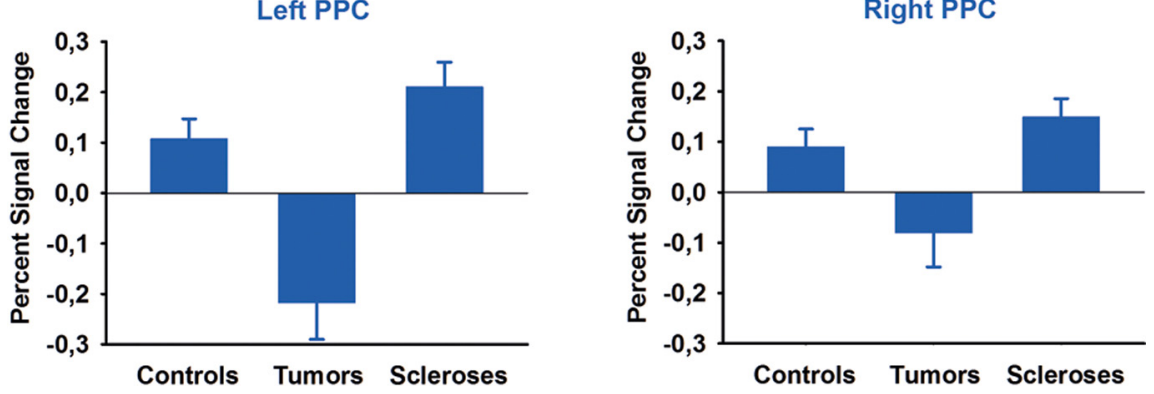

Figure 7. Percentage signal change in neocortical regions. Percentage signal change in DLPFC and PPC ROls for controls, brain tumor patients, and hippocampal sclerosis patients is plotted for illustrational purposes. Bars represent SEM.

lateral hippocampal dysfunction results from complex network reorganization. In patients with normal memory performance, this pattern consisted both of activation of the contralateral hippocampus and of increased recruitment of a widespread shortterm memory network in neocortex.

The approach chosen for our study differs in several respects from previous functional imaging studies on unoperated patients with hippocampal pathology. In most of these studies, ambiguities on the functional status of the diseased hippocampus and a possible contribution of active epilepsy interfered with an unequivocal interpretation of brain activation in the intact hippocampus and extrahippocampal regions (Janszky et al., 
2004; Powell et al., 2007; Figueiredo et al., 2008; Bonelli et al., 2010). The patients investigated here were seizure-free and had postsurgical hippocampal lesions that excluded any residual function within the lesion. In addition, similar anticonvulsive treatment was present in both of our patient groups, rendering pharmacological effects on group differences in fMRI results unlikely (Koepp, 2011). We are thus confident that the correlation between performance and left hippocampal activation observed here results from compensatory reorganization of a memory network that normally depends on integrity of the right hippocampus.

It has been questioned repeatedly whether contralesional hippocampal activation in patients with unilateral hippocampal pathology reflects compensation or if it indicates inefficient reorganization. In the latter view, activity in residual ipsilesional hippocampal structures is supposed to be the main mechanism supporting intact memory (Duncan, 2010). For example, in patients with unoperated unilateral hippocampal sclerosis, greater activation of the diseased hippocampus was associated with better memory performance for verbal and visual stimuli, whereas greater activation of the intact hippocampus was associated with decreased performance (Powell et al., 2007). In addition, changes in functional connectivity between contralateral hippocampus and neocortical regions may not necessarily indicate compensatory mechanisms, but have also been found to reflect pathological brain plasticity (Campo et al., 2012). This contrasts with recent studies showing positive correlations between resting state functional connectivity of the unaffected MTL and performance in memory tasks (Bettus et al., 2009; Doucet et al., 2012). Similarly, it has been observed that patients with unilateral hippocampal sclerosis activate the contralateral MTL during encoding of visual stimuli (Figueiredo et al., 2008). Using multivoxel pattern analysis, activity patterns of specific memories were detected in the contralateral but not in the sclerotic hippocampus (Bonnici et al., 2013). Our findings do not reconcile these divergent results, but do provide strong and direct evidence that recruitment of the contralateral hippocampus in our patients is neither a mere disease-related phenomenon nor a marker of incomplete compensation, but rather an important element of successful reorganization of associative short-term memory. It should be kept in mind that hippocampal sclerosis is not a homogenous entity and that neuronal cell loss in hippocampal subfields is variable (Wyler et al., 1992; Blümcke et al., 2007). It is thus tempting to speculate that some of the discrepancies between our results and previous studies may have been caused by heterogeneous neuropathology that may have differentially modulated the efficiency of functional reorganization processes.

So far, most previous functional imaging studies in patients with hippocampal damage have restricted analysis to regions of interest in the MTLs ipsilateral and contralateral to the lesion side. This approach undoubtedly yields valuable information for treatment of individual patients (Duncan, 2010). However, the activation pattern in our patients with intact memory performance shows that successful memory reorganization with hippocampal damage is more than activation of residual ipsilateral hippocampus or trans-hemispheric shift of function into intact contralateral hippocampus. Consistent with the position of the hippocampus as a densely interconnected hub within brain networks (van den Heuvel and Sporns, 2011), lesion of the hippocampus and subsequent reorganization processes in our patients appear to produce significant nonlocal changes in brain activation that include widely distributed neocortical regions in both hemispheres (Alstott et al., 2009). Compared with patients with deficient performance, patients with intact memory performance showed increased recruitment of a bilateral frontoparietal network that has been reported repeatedly in visual working memory studies (Ranganath and D'Esposito, 2001; Pessoa et al., 2002; Wager and Smith, 2003) and that was also active in our healthy control group. More specifically, activation of corresponding regions in prefrontal and parietal cortex has been found while normal subjects performed short-term memory tasks with delayed match-to-sample designs that required association of visual and spatial information across delays of some seconds (Piekema et al., 2006, 2010; Hannula and Ranganath, 2008). Activity in the left DLPFC moreover correlated with performance in our memory task, thus suggesting that - with the exception of the left hippocampus-reorganization of associative short-term memory mainly occurs by increased recruitment of a preexisting memory network. This complements recent findings from magnetoencephalic recordings in patients with memory disorders after traumatic brain injury, which showed that successful rehabilitation of memory is paralleled by changes in cerebral connectivity profiles that become progressively similar to controls (Castellanos et al., 2010).

The co-occurrence of left hippocampal and bilateral neocortical activation in patients with intact associative short-term memory and the absence of both in patients with deficient performance suggest that these two phenomena may be interdependent. In normal human subjects, synchronization of neural oscillatory activity between hippocampus and neocortex appears to be an important mechanism for maintenance of information across short-term memory delays (Düzel et al., 2010; Fell and Axmacher, 2011; Poch and Campo, 2012). Studies in experimental animals showed that neocortical activity is coordinated by hippocampal activity in the theta range (Jones and Wilson, 2005; Lee et al., 2005). Correspondingly, patients with hippocampal pathology and associative short-term memory deficits have shown a loss of synchrony in the theta range between occipital and temporal brain regions that was selective for an associative memory task (Cashdollar et al., 2009). These findings therefore suggest that neocortical activity during the memory delay of such tasks may depend critically on hippocampal integrity. Although speculative, an extension of this conclusion from our findings would be that enhanced recruitment of an associative short-term memory network in neocortex that compensates for right hippocampal damage may depend on the left hippocampus.

What drives development of the compensatory activation pattern in our patients? Although similar in terms of lesions and most clinical variables, the investigated patient groups differed significantly in the preoperative time course of the underlying disorder. In patients with successful compensation, epilepsy had its onset in childhood or youth, whereas in patients with deficient performance, onset of epilepsy was less than 2 years preoperatively. The current pathophysiological model of hippocampal sclerosis suggests that onset of hippocampal pathology may even precede onset of epilepsy by a latent period of several years (Baulac et al., 2004; Sadler, 2006). Therefore, early onset and total disease duration are factors that may have contributed to or allowed for successful compensation. In addition, evidence from imaging studies in epilepsy patients further suggests that seizure activity may activate processes of compensatory plasticity (Janszky et al., 2004; Figueiredo et al., 2008). Preoperative seizure frequency thus may also have influenced functional compensation in our patients. It remains to be determined whether the observed memory network reorganization is disease specific or if 
it also occurs with other major neurological disorders that lead to focal lesions affecting the MTL, such as herpes encephalitis, amnestic stroke, or traumatic brain injury (Bartsch, 2012). At least for non-focal bilateral diseases affecting the hippocampal formation, such as in Alzheimer's disease and mild cognitive impairment, compensatory recruitment of neocortical regions during memory tasks has been reported (Dickerson and Sperling, 2009; Schwindt and Black, 2009). This is particularly intriguing because this process may provide targets for nonpharmacological neuromodulation techniques in patients with memory disorders (Rossi and Rossini, 2004; Manenti et al., 2012; Nardone et al., 2012).

In summary, we have shown that successful reorganization of a memory function after focal hippocampal damage is achieved by recruitment of an extended network that involves the contralateral hippocampus and widely distributed regions of neocortex in both hemispheres. It appears that for this pattern to develop, residual functional hippocampal structures and the temporal properties of the underlying disease are important prerequisites. Longitudinal studies in patients with focal hippocampal lesions that combine behavioral and imaging measures may clarify whether analysis of memory-related activations will allow for individual prediction of memory reorganization and for corresponding therapeutic interventions in diseases affecting the hippocampus.

\section{References}

Alstott J, Breakspear M, Hagmann P, Cammoun L, Sporns O (2009) Modeling the impact of lesions in the human brain. PLoS Comput Biol 5:e1000408. CrossRef Medline

Altman DG (1991) Practical statistics for medical research. London: Chapman and Hall.

Ashburner J, Friston KJ (2005) Unified segmentation. Neuroimage 26:839851. CrossRef Medline

Bartsch T (2012) The hippocampus in neurological disease. In: The clinical neurobiology of the hippocampus (Bartsch T, ed), pp 200-222. Oxford: Oxford UP.

Baulac S, Gourfinkel-An I, Nabbout R, Huberfeld G, Serratosa J, Leguern E, Baulac M (2004) Fever, genes, and epilepsy. Lancet Neurol 3:421-430. CrossRef Medline

Bettus G, Guedj E, Joyeux F, Confort-Gouny S, Soulier E, Laguitton V, Cozzone PJ, Chauvel P, Ranjeva JP, Bartolomei F, Guye M (2009) Decreased basal fMRI functional connectivity in epileptogenic networks and contralateral compensatory mechanisms. Hum Brain Mapp 30:1580-1591. CrossRef Medline

Blümcke I, Pauli E, Clusmann H, Schramm J, Becker A, Elger C, Merschhemke M, Meencke HJ, Lehmann T, von Deimling A, Scheiwe C, Zentner J, Volk B, Romstöck J, Stefan H, Hildebrandt M (2007) A new clinico-pathological classification system for mesial temporal sclerosis. Acta Neuropathol 113:235-244. CrossRef Medline

Bonelli SB, Powell RH, Yogarajah M, Samson RS, Symms MR, Thompson PJ, Koepp MJ, Duncan JS (2010) Imaging memory in temporal lobe epilepsy: predicting the effects of temporal lobe resection. Brain 133:11861199. CrossRef Medline

Bonnici HM, Sidhu M, Chadwick MJ, Duncan JS, Maguire E a (2013) Assessing hippocampal functional reserve in temporal lobe epilepsy: A multi-voxel pattern analysis of fMRI data. Epilepsy Res pii:S09201211(13)00017-X. CrossRef Medline

Braun M, Finke C, Ostendorf F, Lehmann TN, Hoffmann KT, Ploner CJ (2008) Reorganization of associative memory in humans with longstanding hippocampal damage. Brain 131:2742-2750. CrossRef Medline

Campo P, Garrido MI, Moran RJ, Maestú F, García-Morales I, Gil-Nagel A, del Pozo F, Dolan RJ, Friston KJ (2012) Remote effects of hippocampal sclerosis on effective connectivity during working memory encoding: a case of connectional diaschisis? Cereb Cortex 22:1225-1236. CrossRef Medline

Cashdollar N, Malecki U, Rugg-Gunn FJ, Duncan JS, Lavie N, Duzel E (2009) Hippocampus-dependent and -independent theta-networks of active maintenance. Proc Natl Acad Sci U S A 106:20493-20498. CrossRef Medline
Castellanos NP, Paúl N, Ordóñez VE, Demuynck O, Bajo R, Campo P, Bilbao A, Ortiz T, del-Pozo F, Maestú F (2010) Reorganization of functional connectivity as a correlate of cognitive recovery in acquired brain injury. Brain 133:2365-2381. CrossRef Medline

Castellanos NP, Bajo R, Cuesta P, Villacorta-Atienza JA, Paúl N, GarciaPrieto J, Del-Pozo F, Maestú F (2011) Alteration and reorganization of functional networks: a new perspective in brain injury study. Front Hum Neurosci 5:90. CrossRef Medline

Crinion J, Ashburner J, Leff A, Brett M, Price C, Friston K (2007) Spatial normalization of lesioned brains: performance evaluation and impact on fMRI analyses. Neuroimage 37:866-875. CrossRef Medline

Dickerson BC, Sperling RA (2009) Large-scale functional brain network abnormalities in Alzheimer's disease: insights from functional neuroimaging. Behav Neurol 21:63-75. CrossRef Medline

Doucet G, Osipowicz K, Sharan A, Sperling MR, Tracy JI (2012) Extratemporal functional connectivity impairments at rest are related to memory performance in mesial temporal epilepsy. Hum Brain Mapp 00.

Duncan JS (2010) Imaging in the surgical treatment of epilepsy. Nat Rev Neurol 6:537-550. CrossRef Medline

Düzel E, Penny WD, Burgess N (2010) Brain oscillations and memory. Curr Opin Neurobiol 20:143-149. CrossRef Medline

Engel JJ, Van Ness PC, Rasmussen T, Ojemann LM (1993) Outcome with respect to epileptic seizures. In: Surgical treatment of the epilepsies, Ed 2. (Engel J J, ed), pp 609-621. New York: Raven.

Fell J, Axmacher N (2011) The role of phase synchronization in memory processes. Nat Rev Neurosci 12:105-118. CrossRef Medline

Figueiredo P, Santana I, Teixeira J, Cunha C, Machado E, Sales F, Almeida E, Castelo-Branco M (2008) Adaptive visual memory reorganization in right medial temporal lobe epilepsy. Epilepsia 49:1395-1408. CrossRef Medline

Finke C, Braun M, Ostendorf F, Lehmann TN, Hoffmann KT, Kopp U, Ploner CJ (2008) The human hippocampal formation mediates shortterm memory of colour-location associations. Neuropsychologia 46:614623. CrossRef Medline

Friston K (2012) Ten ironic rules for non-statistical reviewers. Neuroimage 61:1300-1310. CrossRef Medline

Hannula DE, Ranganath C (2008) Medial temporal lobe activity predicts successful relational memory binding. J Neurosci 28:116-124. CrossRef Medline

Hannula DE, Tranel D, Cohen NJ (2006) The long and the short of it: relational memory impairments in amnesia, even at short lags. J Neurosci 26:8352-8359. CrossRef Medline

Horn W (1983) Leistungsprüfsystem. Göttingen: Hogrefe.

Insausti R, Amaral DG (2004) Hippocampal formation. In: The human nervous system (Paxinos G, Mai J, eds), pp 871-915. Amsterdam: Elsevier Academic.

Insausti R, Juottonen K, Soininen $\mathrm{H}$, Insausti AM, Partanen K, Vainio P, Laakso MP, Pitkänen A (1998) MR volumetric analysis of the human entorhinal, perirhinal, and temporopolar cortices. AJNR Am J Neuroradiol 19:659-671. Medline

Janszky J, Ollech I, Jokeit H, Kontopoulou K, Mertens M, Pohlmann-Eden B, Ebner A, Woermann FG (2004) Epileptic activity influences the lateralization of mesiotemporal fMRI activity. Neurology 63:1813-1817. CrossRef Medline

Jeneson A, Squire LR (2012) Working memory, long-term memory, and medial temporal lobe function. Learn Mem 19:15-25. CrossRef Medline

Jenkinson M, Smith S (2001) A global optimisation method for robust affine registration of brain images. Med Image Anal 5:143-156. CrossRef Medline

Jones MW, Wilson MA (2005) Theta rhythms coordinate hippocampalprefrontal interactions in a spatial memory task. PLoS Biol 3:e402. CrossRef Medline

Koepp MJ (2011) Gender and drug effects on neuroimaging in epilepsy. Epilepsia 52:35-37. CrossRef Medline

Lee H, Simpson GV, Logothetis NK, Rainer G (2005) Phase locking of single neuron activity to theta oscillations during working memory in monkey extrastriate visual cortex. Neuron 45:147-156. CrossRef Medline

Lehrl S (2005) Mehrfachwahl-Wortschatz-Intelligenztest. Göttingen: Hogrefe.

Macmillan NA, Creelman CD (2005) Detection theory. Mahwah: Lawrence Erlbaum Associates.

Manenti R, Cotelli M, Robertson IH, Miniussi C (2012) Transcranial brain stimulation studies of episodic memory in young adults, elderly adults 
and individuals with memory dysfunction: a review. Brain Stimul 5:103109. CrossRef Medline

Nardone R, Bergmann J, Christova M, Caleri F, Tezzon F, Ladurner G, Trinka E, Golaszewski S (2012) Effect of transcranial brain stimulation for the treatment of Alzheimer disease: a review. Int J Alzheimers Dis 2012: 687909. CrossRef Medline

Olson IR, Page K, Moore KS, Chatterjee A, Verfaellie M (2006) Working memory for conjunctions relies on the medial temporal lobe. J Neurosci 26:4596-4601. CrossRef Medline

Pessoa L, Gutierrez E, Bandettini P, Ungerleider L (2002) Neural correlates of visual working memory: fMRI amplitude predicts task performance. Neuron 35:975-987. CrossRef Medline

Piekema C, Kessels RP, Mars RB, Petersson KM, Fernández G (2006) The right hippocampus participates in short-term memory maintenance of object-location associations. Neuroimage 33:374-382. CrossRef Medline

Piekema C, Rijpkema M, Fernández G, Kessels RP (2010) Dissociating the neural correlates of intra-item and inter-item working-memory binding. PLoS One 5:e10214. CrossRef Medline

Poch C, Campo P (2012) Neocortical-hippocampal dynamics of working memory in healthy and diseased brain states based on functional connectivity. Front Hum Neurosci 6:36. CrossRef Medline

Powell HW, Richardson MP, Symms MR, Boulby PA, Thompson PJ, Duncan JS, Koepp MJ (2007) Reorganization of verbal and nonverbal memory in temporal lobe epilepsy due to unilateral hippocampal sclerosis. Epilepsia 48:1512-1525. CrossRef Medline

Ranganath C (2010) A unified framework for the functional organization of the medial temporal lobes and the phenomenology of episodic memory. Hippocampus 20:1263-1290. CrossRef Medline

Ranganath C, D’Esposito M (2001) Medial temporal lobe activity associated with active maintenance of novel information. Neuron 31:865-873. CrossRef Medline

Rorden C, Karnath HO, Bonilha L (2007) Improving lesion-symptom mapping. J Cogn Neurosci 19:1081-1088. CrossRef Medline

Rossi S, Rossini PM (2004) TMS in cognitive plasticity and the potential for rehabilitation. Trends Cogn Sci 8:273-279. CrossRef Medline

Sadler RM (2006) The syndrome of mesial temporal lobe epilepsy with hippocampal sclerosis: clinical features and differential diagnosis. Adv Neurol 97:27-37. Medline

Schwindt GC, Black SE (2009) Functional imaging studies of episodic memory in Alzheimer's disease: a quantitative meta-analysis. Neuroimage 45: 181-190. CrossRef Medline

Smith SM, Jenkinson M, Woolrich MW, Beckmann CF, Behrens TE, Johansen-Berg H, Bannister PR, De Luca M, Drobnjak I, Flitney DE, Niazy RK, Saunders J, Vickers J, Zhang Y, De Stefano N, Brady JM, Matthews PM (2004) Advances in functional and structural MR image analysis and implementation as FSL. Neuroimage 23:S208-S219. CrossRef Medline

Stretton J, Winston G, Sidhu M, Centeno M, Vollmar C, Bonelli S, Symms M, Koepp M, Duncan JS, Thompson PJ (2012) Neural correlates of working memory in Temporal Lobe Epilepsy - An fMRI study. Neuroimage 60:1696-1703. CrossRef Medline

van den Heuvel MP, Sporns O (2011) Rich-club organization of the human connectome. J Neurosci 31:15775-15786. CrossRef Medline

Wager TD, Smith EE (2003) Neuroimaging studies of working memory: a meta-analysis. Cogn Affect Behav Neurosci 3:255-274. CrossRef Medline

Wyler AR, Curtis Dohan F, Schweitzer JB, Berry AD (1992) A grading system for mesial temporal pathology (hippocampal sclerosis) from anterior temporal lobectomy. Journal of Epilepsy 5:220-225. CrossRef 